Methods Human neutrophils were purified from healthy volunteers by discontinuous percoll gradients. PAO-1 and PHD-deficient strains were grown in lysogeny broth and equivalent growth curves confirmed. Supernatants from wild-type and PHD-deficient $P$. aeruginosa were harvested at two hours and then co-cultured with neutrophils in normoxia and hypoxia. Neutrophil viability and apoptosis was then assessed using AnnexinV/To-pro3 staining on flow cytometry at three and five hours.

Results Control neutrophils in normoxia saw a decrease in viability of $6 \%$ between three and five hours. Neutrophils treated with supernatant from the PHD-deficient strain experienced a decrease in viability from 3139 ( \pm 968 ) cells at three hours to $2058( \pm 586)$ at five hours - a decline of 34\% ( $\mathrm{P}<0.05)$. Normoxic neutrophils treated with the wild-type strain, however, saw a decrease of $21 \%(\mathrm{P}<0.05)$.

Hypoxic conditions reversed the killing effects of wild-type $P$. aeruginosa: after five hours neutrophils in normoxia experienced a $21 \%$ decrease in viability, whereas the viability of hypoxic cells only decreased by $8 \%(\mathrm{P}<0.05)$.

Discussion These data highlight the relationship between tissue oxygen tensions and host immunity and that bacteria have evolved virulence factors with novel mechanisms of action; namely preventing neutrophil survival at sites of inflammation. Moreover, the potential oxygen-sensing capabilities of prokaryotes are intricately linked to bacterial virulence.

\section{S45 EVALUATING THE SENSITIVITY AND SPECIFICITY OF ACTIVE NEUTROPHIL ELASTASE AS A BIOMARKER FOR BACTERIAL INFECTION IN SUBJECTS WITH COPD}

\author{
${ }^{1} \mathrm{~S} J$ Thulborn, ${ }^{2} \mathrm{~N}$ Akram, ${ }^{3} \mathrm{~V}$ Mistry, ${ }^{3} \mathrm{CE}$ Brightling, ${ }^{4} \mathrm{~K}$ Moffitt, ${ }^{4} \mathrm{D}$ Ribeiro, ${ }^{1} \mathrm{M}$ Bafadhel. \\ ${ }^{1}$ University of Oxford, Oxford, UK; ${ }^{2}$ Oxford Brookes University, Oxford, UK; ${ }^{3}$ University of \\ Leicester, Leicester, UK; ${ }^{4}$ ProAxsis Ltd, UK, Carterton
}

\subsection{6/thoraxjnl-2016-209333.51}

Introduction COPD is a neutrophilic disease, with the majority of subjects having a sputum neutrophil percentage of $>60 \%$. Neutrophil elastase (NE) is a serine proteinase, secreted by neutrophils and macrophages during inflammation and has a role in the destruction of bacteria within the host. New advancements now allow accurate assessment of active protease levels in complex biological samples. We sought to investigate if active NE could be used as a biomarker for bacterial infection in subjects with COPD.

Methods NE was quantified using ProteaseTag ${ }^{\text {TM }}$ active NE Immunoassay (ProAxsis, Belfast) from cell-free sputum supernatant from 31 COPD subjects (20 Males; mean age 65, range 45 to 81$)$ at stable state and during an exacerbation. Bacterial infection was defined as $\geq 10^{7} \mathrm{CFU} / \mathrm{mL}$ in sputum. Subject demographics, sputum cell differential counts and polymerase chain reaction (PCR) for respiratory pathogens were measured.

Results Active NE was higher during an exacerbation compared to stable state (fold difference (95\% CI) 0.50 (0.22 to 0.78), $\mathrm{p}=0.001$ ) (Figure 1). NE correlated with total sputum neutrophils ( $\mathrm{p}<0.0001, \mathrm{r}=0.48$ ) and total bacterial load measured by $\mathrm{CFU} / \mathrm{mL}(\mathrm{p}<0.01, \mathrm{r}=0.39)$ and $\mathrm{qPCR}(\mathrm{p}<0.05, \mathrm{r}=0.33)$. When looking at the main respiratory pathogens no correlations were seen between $H$. influenzae ( $\mathrm{p}=0.43, \mathrm{r}=-0.11$ ), S. aureus $(\mathrm{p}=0.34, \mathrm{r}=-0.14)$ or $\mathrm{S}$. pneumonia $(\mathrm{p}=0.11$, $\mathrm{r}=0.23$ ); however a correlation was seen between NE and $M$. catarrhalis ( $\mathrm{p}=0.01, \mathrm{r}=0.36$ ). NE has an area under the receiver operator curve of 0.72 [0.58 to 0.85$]$ to identify a bacterial infection with a sensitivity and specificity of $67.74 \%$ and $67.86 \%$ at a NE cut off of $2335 \mathrm{ng} / \mathrm{mL}$.

Conclusion Active NE is elevated during a COPD exacerbation compared to baseline. Active NE is associated with neutrophilic inflammation and bacteria; and may be a viable biomarker for bacterial infection in COPD.

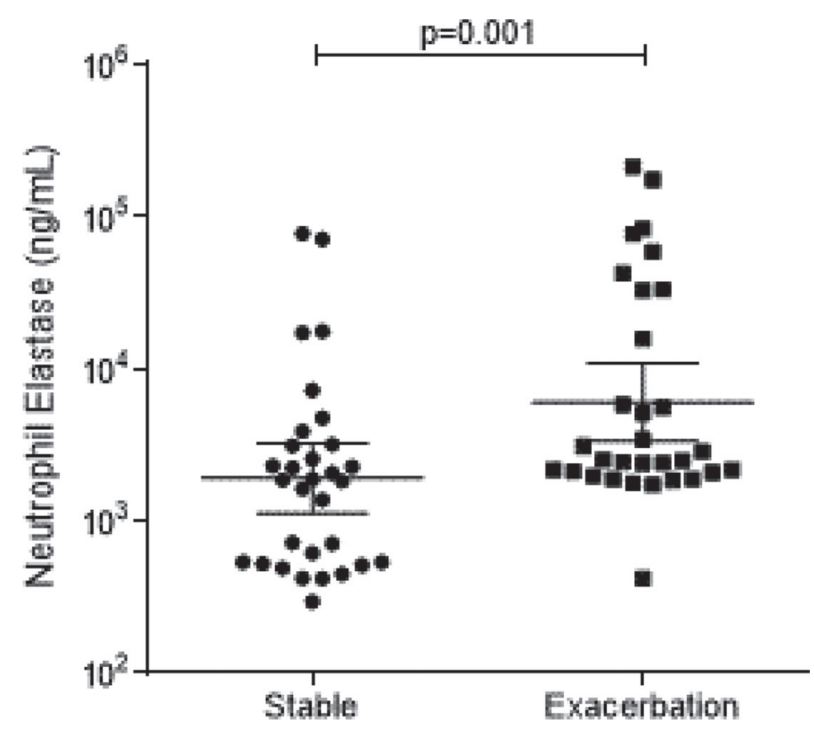

Abstract S45 Figure 1 Sputum active NE levels at stable and exacerbation state from 31 paired COPD subjects. Mean and $95 \% \mathrm{Cl}$

\section{S46 NEUTROPHIL VASCULAR ENDOTHELIAL GROWTH FACTOR (VEGF) AS A DRIVING FORCE FOR ANGIOGENESIS IN BRONCHIECTASIS?}

${ }^{1} \mathrm{CC}$ Cole, ${ }^{2} \mathrm{SC}$ Carnell, ${ }^{3} \mathrm{KJ}$ Jiwa, ${ }^{4} \mathrm{JB}$ Birch, ${ }^{1} \mathrm{KH}$ Hester, ${ }^{1} \mathrm{CW}$ Ward, ${ }^{1} \mathrm{JS}$ Simpson, ${ }^{2} \mathrm{ADS}$ De Soyza. Institute of Cellular Medicine, Newcastle University, Newcastle Upon Tyne, UK; ${ }^{2}$ Newcastle Clinical Trials Unit, Newcastle Upon Tyne, UK; ${ }^{3}$ Sir William Leech Centre for Lung Research, Freeman Hospital, Newcastle Upon Tyne, UK; ${ }^{4}$ Institute for Cell and Molecular Biosciences, Newcastle Upon Tyne, UK

\subsection{6/thoraxjnl-2016-209333.52}

Introduction Bronchiectasis (BR) in a pulmonary disease thought to involve a characteristic dilation of the bronchi resulting from a cycle of airway infection and inflammation. This inflammation is believed to be driven by neutrophils, which are present in the BR lung in high number. Vascular endothelial growth factor (VEGF) is a pro-angiogenic cytokine that may be upregulated in BR and could contribute towards creating a pro-angiogenic airway environment by supporting neutrophil migration into the airway tissue, however this has yet to be shown.

Aims 1) Examine the BR airway for any indications of increased angiogenesis, 2) Assess the ability of neutrophils to secrete VEGF upon stimulation in vitro, 3) Evaluate sera/sputa samples VEGF concentration to determine if VEGF could act as a biomarker for BR severity.

Methods Healthy volunteer (HV) and BR endobronchial biopsies were stained with a HRP conjugated anti-CD31 antibody, allowing blood vessels to be counted in a blinded manner. Peripheral blood neutrophils isolated from HV were stimulated (e.g. with TNF- $\alpha$ or bacterial PAMPs) for 4 hours, VEGF levels in supernatants were then quantified using ELISA. A VEGF ELISA was also used to determine VEGF concentration in sera and sputa samples 
from BR patients $(\mathrm{n}=115)$, categorised by bronchiectasis severity index (BSI) scores and sera samples from $\mathrm{HV}$ controls $(\mathrm{n}=26)$

Results Endobronchial biopsies from BR airways had a significantly ( $p<0.05$ ) higher number of blood vessels per $\mathrm{mm}$ of basement membrane than HV samples (18 and 9 blood vessels/ $\mathrm{mm}$ basement membrane respectively). Stimulation of HV neutrophils with a variety of molecules (PMA, fMLP, LPS, TNF- $\alpha$ etc.) resulted in a significant increase in VEGF secretion compared to unstimulated $(\mathrm{p}<0.05)$. Although elevated VEGF was found in some patient samples there was no significant correlation between sera/sputa VEGF and individual patient BSI scores.

Conclusion The increased presence of vascular tissue seen in BR could indicate a pro-angiogenic airway environment in BR. The in vitro data collected also show that a variety of stimulants can initiate secretion of VEGF by neutrophils. However, our data does not suggest that VEGF levels in sera or sputa can be used to predict disease severity.

\section{S47 PNEUMOLYSIN PROMOTES NEUTROPHIL: PLATELET AGGREGATION IN VITRO}

${ }^{1} \mathrm{JG} \mathrm{Nel,}{ }^{1} \mathrm{C}$ Durandt, ${ }^{1} \mathrm{AJ}$ Theron, ${ }^{1} \mathrm{GR}$ Tintinger, ${ }^{2} \mathrm{TJ}$ Mitchell, ${ }^{3} \mathrm{C}$ Feldman, ${ }^{1} \mathrm{R}$ Anderson. ${ }^{1}$ University of Pretoria, Pretoria, South Africa; ${ }^{2}$ University of Birmingham, Birmingham, UK; ${ }^{3}$ University of the Witwatersrand, Johannesburg, South Africa

\subsection{6/thoraxjnl-2016-209333.53}

Introduction and objectives The pneumococcal cholesterol-binding, pore-forming toxin, pneumolysin (Ply), appears to be a key mediator not only of the acute lung injury, but also myocardial damage, associated with severe pneumococcal disease. Although direct Ply-mediated cardiopulmonary toxicity has been implicated, the neutrophil- and platelet-targeted pro-inflammatory activities of the toxin are also believed to contribute to the pathogenesis of these adverse events, albeit by poorly characterised mechanisms. To test the hypothesis that Ply promotes neutrophil: platelet networking, we have investigated the effects of the toxin on the induction of heterotypic aggregation of these cells in vitro. Methods Neutrophil: platelet-enriched buffy coat suspensions were prepared from the heparinised blood of healthy, adult humans by sedimentation (at $37^{\circ} \mathrm{C}$ ) and diluted 1:50 in Hanks' balanced salt solution. Following $5 \mathrm{~min}$ of preincubation, recombinant Ply (10-80 ng/ml), or the pneumolysoid, delta 6Ply (attenuated with respect to pore-forming activity, negative control), or adenosine 5'-diphosphate (ADP, $100 \mu \mathrm{M}$, positive control) were added to the cell suspensions. After a further $5 \mathrm{~min}$ period of incubation at $37^{\circ} \mathrm{C}$, samples were stained with $5 \mu \mathrm{l}$ of each of the following murine, anti-human, fluorochrome-labelled monoclonal antibodies: CD16-APC (neutrophils), CD42a-PE (platelets), and CD45-Krome Orange, and incubated for $15 \mathrm{~min}$ at room temperature in the dark. This was followed by analysis of samples at a slow rate using a Gallios flow cytometer. The relative numbers of platelets interacting with a single neutrophil were determined using the relative mean fluorescence intensities of CD16+/CD42a+/CD45+ neutrophils.

Results These are shown in the accompanying table. Addition of Ply to the mixed cell suspension resulted in statistically significant dose-related formation of neutrophil:platelet aggregates which was maximal at $80 \mathrm{ng} / \mathrm{ml}$ and greater in magnitude to that observed with ADP, while delta6Ply was ineffective.

Conclusion Ply, at pathologically-relevant concentrations, promotes neutrophil:platelet aggregation in vitro, an activity which is dependent on the pore-forming properties of the toxin. Given the increasing recognition of the role played by platelets in driving neutrophilic inflammation, this activity of Ply may exacerbate pulmonary and myocardial injury in severe pneumococcal disease.

$\begin{aligned} & \text { Abstract S47 Table } 1 \\
& \text { neutrophil: platelet aggregates }\end{aligned}$
\begin{tabular}{ll} 
System & $\begin{array}{l}\text { Neutrophil: platelet aggregates } \\
\text { (mean fluorescence intensity } \pm \text { SDs) }\end{array}$ \\
\hline Background & $11.24 \pm 5.0$ \\
ADP $(100 \mu \mathrm{M})$ & $20.63 \pm 5.6^{*}$ \\
Ply $(10 \mathrm{ng} / \mathrm{ml})$ & $18.90 \pm 10.7^{*}$ \\
Ply $(20 \mathrm{ng} / \mathrm{ml})$ & $27.92 \pm 16.08^{*}$ \\
Ply $(40 \mathrm{ng} / \mathrm{ml})$ & $33.21 \pm 17.09^{*}$ \\
Ply $(80 \mathrm{ng} / \mathrm{ml})$ & $42.09 \pm 23.05^{*}$ \\
Delta6Ply $(80 \mathrm{ng} / \mathrm{ml})$ & $14.86 \pm 7.49$ \\
\hline${ }^{*} p<0.05-p<0.0009$ &
\end{tabular}

\section{S48 TARGETING SIGLECS TO REDUCE PROTEASE-MEDIATED DESTRUCTION IN TUBERCULOSIS}

W Beynon, R McMullan, D McAuley, C O'Kane. Centre for Experimental Medicine, Queen's University, Belfast, UK

\subsection{6/thoraxjn--2016-209333.54}

Background Tuberculosis (TB) is an inflammatory disease caused by infection with Mycobacterium tuberculosis (Mtb). The disease is often characterised by destructive pulmonary pathology, which itself aids transmission, and many who complete otherwise successful treatment are left with lasting respiratory impairment following TB-driven tissue damage and remodelling. This is largely mediated by matrix metalloproteinases (MMP) induced in the inflammatory response.

The CD33-related siglecs are transmembrane receptors that bind sialic acid. They are selectively expressed on immune cells, where they mediate inhibitory signalling. Murine Siglec-E is upregulated on macrophages by LPS. Its activation by crosslinking with sialylated nanoparticles reduces inflammatory cytokine release and mortality in murine models of sepsis and lung injury. Siglecs $-5,-7$ and -9 are candidate human orthologs of SiglecE, known to inhibit inflammatory cell activation and proliferation. We hypothesised that the human orthologs of Siglec-E are upregulated in response to Mtb, which like LPS is a TLR-4 ligand, and that their activation would reduce TB-driven inflammation.

Methods Siglec expression at gene and protein level on primary monocytes isolated from blood donation, and in a monocyte derived macrophage (MDM) model was investigated by qPCR, flow cytometry and western blotting in both unstimulated and Mtb-infected cells. Monocytes and MDMs were infected with $\mathrm{Mtb}$ and incubated with antibodies to either neutralise or crosslink siglecs -7 and -9 . The effect on their secretion of inflammatory cytokines and MMPs or their inhibitors (Tissue Inhibitors of Metalloproteinases - TIMPs) was measured by ELISA.

Results

Siglecs $-5,-7$ and -9 are constitutively expressed on human monocytes and MDMs.

Unlike Siglec-E in mice, these siglecs are not upregulated by LPS stimulation, nor by infection with Mtb. 